\title{
REFLEXIONES SOBRE LA ATENCIÓN DE LOS MENORES CON TRASTORNO DE ESPECTRO AUTISTA EN LOS CENTROS EDUCATIVOS.
}

\author{
Javier Cortés Moreno \\ Eva María Sotomayor Morales \\ Universidad de Jaén (España) \\ Enrique Pastor Seller \\ Universidad de Murcia (España)
}

\begin{abstract}
Resumen. En el ámbito educativo, la inserción de los/as menores con Trastorno de Espectro Autista (TEA) en condiciones óptimas de inclusión resulta, con frecuencia, una tarea compleja. ¿Cuentan los centros educativos con una infraestructura sólida de recursos y de herramientas inclusivas? ¿Disponen de profesionales competentes y especializados en la atención del alumnado con discapacidad?. El presente artículo, pretende responder a estas cuestiones y tiene como objetivo conocer la opinión de expertos/as en TEA sobre la situación y atención que reciben estos/as menores en los centros educativos. Entre las variables analizadas están las tipologías de los centros, las modalidades del Sistema Educativo Español, las herramientas y métodos de inclusión y el rol de los actores de la comunidad educativa (familiares, menores, equipos de orientación educativa, profesorado, profesionales especializados, etc.). La metodología que se ha empleado ha sido cualitativa, extrayendo los datos mediante la realización de dos grupos de discusión en la provincia de Jaén y en Salamanca. La muestra, se ha establecido mediante muestreo intencional, siendo los/as participantes diez profesionales expertos/as en TEA de distintas disciplinas (educación, psicología, trabajo social, terapia ocupacional, logopedia, audición y lenguaje). Todos/as los/as profesionales coinciden como principal resultado que resulta insuficiente la atención especializada que ofrecen los centros escolares, por lo que los/as menores deben complementarla con el apoyo externo del ámbito privado y/o de las asociaciones. De todo ello, se puede concluir que es necesario ampliar la plantilla de profesionales en los centros educativos, así como su formación y especialización en TEA.
\end{abstract}

Palabras clave: Trastorno de Espectro Autista, Centros Educativos, Inclusión, Grupo de Discusión.

\section{CONSIDERATIONS ABOUT THE CARE OF MINORS WITH AUTISTIC SPECTRUM DISORDER IN EDUCATIONAL CENTERS.}

\begin{abstract}
In the educational field, insertion of minors with Autistic Disorder Spectrum (ASD) under optimal inclusion conditions, is a very complex action. Have the educational centers a solid infrastructure of resources and inclusive tools? Do they have competent and specialized professionals in the attention of students with disabilities?.This article, answers these questions and aims to know the opinion of experts about the care that minors receive in the educational centers. The variables analyzed are: tipologies of the centers, modalities of the Spanish Educational System, the tools and methods of inclusion and the role of actors of the educational community (relatives, minors, teams of educational guidance, teaching staff, specialized professionals, etc.). The methodology used has been qualitative. The data were obtained through two discussion groups in Jaen and in Salamanca. The sample has been established through intentional sampling and ten professionals expert in autism of different disciplines have taken part (education, psychology, social work, occupational therapy, speech, language and hearing therapy). As a main result, all professionals agree that the specialized care of the school centers is insufficient, and that
\end{abstract}


is why minors need private external support or partnerships. It is possible to unanimously conclude, that it is necessary to increase the professional staff in the educational centers, as well as their training and specialization in autism.

Keywords: Autism Spectrum Disorders, Educational Centers, Inclusion, Discussion groups.

\title{
REFLEÇÕES SOBRE A ATENÇÃO DE CRIANÇAS COM TRANSTORNO DO ESPECTRO AUTISTA NAS ESCOLAS.
}

\begin{abstract}
Resumo. No ambiente educacional, a inclusão de crianças com Transtorno do Espectro Autista (TEA) em condições ideais de inclusão é uma ação muito complexa. Têm as escolas uma infra-estrutura sólida de recursos e ferramentas inclusivas? Dispõem de profissionais competentes e especializados na atenção de alunos com incapacidade?.Este artigo, responde a estas perguntas e tem como objetivo conhecer a opinião dos especialistas sobre o cuidado recebido pelas crianças nas escolas. As variáveis analisadas são: as tipologías das escolas, as modalidades do Sistema Educacional espanhol, as ferramentas e métodos de inclusão e o papel dos intervenientes da comunidade educacional (família, crianças, trabalho social, terapia ocupacional, professores, profissionais especializados, etc.). A metodologia utilizada foi qualitativa. Os dados foram obtidos por dois grupos de discussão em Jaén e em Salamanca. A amostra foi establecida por meio de amostragem intencional e participaram dez especialistas profissionais em Transtorno do Espectro Autista de disciplinas diferentes (educação, psicologia, trabalho social, terapia ocupacional, logopedia, audição e língua). Como resultado principal, todos os profissionais coincidem em que é insuficiente a atenção especializada das escolas, e é por isso que as crianças precisam de aulas de apoio fora da escola. Pode-se concluir por unanimidade, que as escolas precisam contratar mais profissionais, assim como melhorar a sua formação e especialização em Transtorno do Espectro Autista.
\end{abstract}

Palavras-chave: Transtorno do Espectro Autista, Escolas, Inclusão, Grupos de discussão.

\section{Introducción}

El TEA, según los criterios diagnósticos del manual DSM-V (2013), se caracteriza por presentar déficits persistentes en la comunicación social y en la interacción social, teniendo desde el periodo de desarrollo temprano patrones repetitivos y restringidos de conductas, actividades, e intereses. Estos síntomas causan ciertas alteraciones clínicamente significativas a nivel social, ocupacional o en otras áreas importantes del funcionamiento.

En la actualidad, el TEA es cada vez más común en nuestra sociedad, pues como constatan diversos estudios hay un aumento considerable de los casos detectados y diagnosticados. Autism Society of America (2011) estima que actualmente cerca de 1.500.000 personas en Estados Unidos padecen de autismo. Si se extrapola esta cifra a España, puesto que no existe una estadística oficial de la población española afectada, resultaría una población de más de 250.000 con TEA.

Esta investigación se ha centrado en analizar la atención que reciben los menores con TEA en los centros educativos. Con plena conciencia de que una detección y diagnóstico temprano del trastorno influye considerablemente en la atención escolar, en una primera fase de esta investigación se ha ejecutado otro estudio que analizó el proceso de detección y diagnóstico que habían experimentado treinta casos de familiares con menores con TEA, con el fin de reflejar las distintas dificultades que experimentaron para detectar y diagnosticar el trastorno. 
La detección precoz de este trastorno constituye un factor clave para intervenir adecuadamente en el ámbito educativo e igualmente para mejorar la adaptabilidad y la inclusión del/a menor con TEA en la sociedad. La vulnerabilidad de este colectivo radica principalmente en la heterogeneidad y complejidad del trastorno que dificulta la detección temprana y un diagnóstico preciso. El Instituto de Salud Carlos III (2004), demostró que la familia es la primera en sospechar el desarrollo de su hijo cuando éste tiene un año y diez meses. En este momento es cuando los familiares suelen preocuparse por la situación de su menor e inician un proceso de consultas a distintos profesionales y organismos. La Federación de Autismo de Castilla y León (2006) afirma que un $62,5 \%$ de las familias tienen dificultades en la realización del diagnóstico. Aunque Attwood (1993) apunta que los TEA pueden ser diagnosticados en menores de dieciocho meses pero en la práctica esto puede ser difícil de lograr, debido a la naturaleza del desorden y también a la falta de conocimiento. Según Hernández, J. M y cols. (2005) en la mayoría de las ocasiones se accede a un diagnóstico final a los dos años y medio después de haber iniciado las consultas.

Teniendo en cuenta estos datos previos, considerados como factores influyentes en la atención e inclusión de los menores con TEA en los Centros educativos, con esta investigación se pretende conocer la situación y atención que reciben estos/as menores en los centros educativos, a través de las opiniones que se han podido extraer del discurso, de las experiencias y de las reflexiones de los/as profesionales expertos/as en TEA. Principalmente se hace hincapié en analizar distintas variables como son las tipologías de los centros escolares, las modalidades del Sistema Educativo Español, las herramientas y métodos de inclusión y el rol que juegan los actores de la comunidad educativa (familiares, menores, equipos de orientación educativa, profesorado, profesionales especializados, etc.).

Contextualizando esta investigación a través de otros estudios e investigaciones relacionadas, encontramos que en el ámbito educativo, según Hernández, J.M. y cols. (2005) existe una situación desigual en todo el territorio español según el grado de implantación de servicios educativos en la etapa infantil, de forma que se asegura una mayor detección del TEA donde se han desarrollado las escuelas infantiles y los equipos de atención temprana.

Por otro lado, la Ley Orgánica de 2/2006, de 3 de Mayo, de Educación, establece que la etapa infantil es de carácter voluntario, por tanto si se opta libremente por no escolarizar a un menor, en caso de existir alguna discapacidad puede estar retrasando la detección y su tratamiento.

Estas dos características a nivel general del sistema educativo español, repercuten con diversas consecuencias a los/as menores con discapacidad, pues la falta de recursos para el diagnóstico del TEA, así como las excepciones en las normativas legislativas supone una dificultad para delimitar los criterios de inclusión en los centros educativos y en las estrategias de aprendizaje. El Consorcio de Autismo (2011) expuso que el ámbito educativo es donde más carencias existen y una correcta intervención es fundamental ya que es un elemento decisivo en el pronóstico a largo plazo y en el desarrollo personal y en la autonomía de la persona en el futuro.

Las primeras dificultades de un/a menor con TEA en un centro educativo surgen en el momento de su escolarización. Cuando un centro recibe una solicitud de matrícula de alumnado con discapacidad, debe iniciar con la administración educativa correspondiente un procedimiento de identificación precoz de las necesidades 
educativas específicas del menor, y con ello asegurarse los recursos necesarios que garanticen la atención integral.

En el caso de la Comunidad Autónoma de Andalucía, esto se traduce en una evaluación psicopedagógica; realizada por los Equipos de Orientación Educativa en colaboración con el profesorado y los familiares. Todo/a menor con discapacidad debe poseer un dictamen de escolarización realizado por los Equipos de Orientación Educativa ya que en este documento se reflejara cuáles son sus necesidades educativas especiales, las alternativas de ayudas, apoyos, adaptaciones del alumnado y una propuesta de la modalidad de escolarización más adecuada.

Para la decisión sobre la modalidad de escolarización; según la Ley 9/1999, de 18 de noviembre de Solidaridad en la Educación; se tendrá en cuenta siempre la opinión de los representantes legales del alumnado, inclusive en la elección del centro docente para su escolarización, teniendo prioridad entre aquéllos que estén ubicados en su entorno y que reúnan los recursos personales y materiales más adecuados que garanticen la atención educativa integral, de acuerdo con el dictamen de escolarización y los criterios generales establecidos para la admisión del alumnado. Esto supone que el centro debe de ofrecer profesorado especializado en necesidades educativas especiales, que empleen el uso de las nuevas tecnologías entre sus materiales didácticos elaborados y la utilización de equipamiento técnico específico, como los sistemas de comunicación aumentativa y alternativa.

Se debe dar prioridad a escolarizar al menor en los centros educativos ordinarios, en sus diversas modalidades (grupo ordinario a tiempo completo, grupo ordinario con apoyos en períodos variables y aula de educación especial) para garantizar el mayor grado de integración. Sólo se matriculará en centros específicos de educación especial cuando, por sus especiales características o grado de discapacidad, sus necesidades educativas no puedan ser satisfechas en régimen de integración. En algunos casos se puede contemplar la escolarización combinada en un centro ordinario y un centro especial. Cuando la respuesta educativa requiera equipamiento singular o la intervención de profesionales de difícil generalización para garantizar una oferta educativa sectorizada de escolarización del alumnado con TEA, las Delegaciones Territoriales podrán especializar determinadas aulas específicas de educación especial en centros de educación primaria o educación secundaria.

Rodríguez, I. R., Moreno, F. J. y Aguilera, A. (2007) realizaron un estudio sobre la atención educativa del alumnado con trastorno de espectro autista en la provincia de Sevilla, analizaron la escolarización de este colectivo y describieron las necesidades educativas. Encontraron que de los 165 alumnos/as con TEA de la muestra global había un $52 \%$ que acudía a centros de educación especial, mientras que el $48 \%$ restante estaba escolarizado en centros ordinarios. Detectaron que a medida que los alumnos con TEA crecen, su escolarización se suele distanciar de los ámbitos más inclusivos, pues observaron un mayor número de alumnos/as escolarizados/as en centros ordinarios entre los 6 y los 12 años, y en centros específicos a partir de los 12 años de edad.

En cuanto a los apoyos que reciben los/as menores con TEA en los centros educativos de Sevilla, encontraron que un $73,2 \%$ de la muestra recibía estos apoyos en las aulas especiales. La atención en estas aulas, variaba dependiendo del menor, entre 1 hora y 36 horas semanales. Un 40,6\% de los/as menores recibía sesiones de logopeda o de audición y lenguaje, entre media hora o un máximo de 5 horas semanales. Igualmente solo el 37\% del alumnado recibía el apoyo de un educador o monitor. Con respecto al apoyo de un psicomotricista, un $80,4 \%$ de la muestra carece de esta atención 
y aquellos/as menores que lo reciben, en su mayoría, es en centros educativos específicos. En el caso de la atención del servicio de psicología, solo lo recibía un 14\% de los/as menores, siendo el mínimo una hora semanal y el máximo ocho horas semanales.

Entre otros datos relevantes de este estudio, cabe destacar el diagnóstico de los/as menores, el cual era conocido por parte del profesorado en un 97,8\% de los casos. El diagnóstico se lo proporcionó, en su mayoría la asociación/institución a la que pertenecen los menores con TEA, en concreto en un $28,1 \%$ y en otros casos, los facilitaban al centro los padres y madres, los orientadores del centro educativo o los Equipos de Orientación Educativa. Por otro lado, este estudio señala, que solamente el $30 \%$ del alumnado dispone de Adaptaciones Curriculares Individualizadas (ACI).

\section{Método}

La metodología empleada en la investigación sobre la atención que reciben los/as menores con TEA en el ámbito educativo por parte del sistema, de los/as profesionales, de los/as expertos/as y de los demás agentes de la comunidad educativa, se ha basado en la realización de varios grupos de discusión con aquellos profesionales que tienen contacto con menores con TEA.

Los grupos de discusión se consideran una de las múltiples técnicas cualitativas de recolección de información y de datos sobre un fenómeno. Huertas E. y Vigier F. (2010) definen esta herramienta cualitativa como la reunión de un grupo de personas que poseen ciertas características comunes, guiada por un moderador y diseñada con el objetivo de obtener información sobre un tema específico en un espacio y un tiempo determinado. En esta investigación que se presenta, el principal objetivo de utilizar esta técnica ha sido conseguir información en profundidad, valiosa y que sirva para ilustrar la situación actual en cuanto a la atención que reciben los/as menores con TEA en los centros educativos. Del mismo modo, con el discurso y la opinión de los/as expertos en TEA se pueden identificar los aspectos claves para mejorar la atención educativa para este colectivo.

La parte fundamental e imprescindible de esta investigación, sin lugar a dudas, lo constituye el diseño del grupo de discusión. Siguiendo las seis fases de Suarez (2005) se ha diseñado una planificación flexible y que sirvió de referencia para guiar el proceso.

En la primera fase, se ha realizado una revisión exhaustiva de la literatura existente sobre el tema objeto de estudio. Con esta fase exploratoria, se han podido definir con precisión los objetivos generales que se pretenden alcanzar con la realización del grupo de discusión.

Siendo importante conocer la opinión y experiencias que tienen los/as expertos/as en TEA sobre la situación y atención que reciben estos/as menores en los centros educativos y cómo influye el hecho de disponer de un diagnóstico precoz del trastorno en el proceso de enseñanza.

La segunda fase, consistía en la preparación del planteamiento global de los contenidos que se abordarían en el grupo de discusión. Asimismo, se decidió el número total de grupos de discusión a realizar y los participantes de cada uno de ellos. Se 
organizaron dos grupos de discusión, que se realizaron en la provincia de Jaén y en Salamanca.

A continuación, se detalla el esquema de contenidos con los temas centrales y específicos que sirvieron al moderador como guion para el abordaje y desarrollo de los grupos de discusión realizados.

Tabla1

Guion del Grupo de Discusión

\begin{tabular}{|c|c|}
\hline TEMAS CENTRALES & TEMAS ESPECÍFICOS \\
\hline La Detección Temprana del TEA & $\begin{array}{l}\text { - } \text { Sospechas del TEA. } \\
\text { - El proceso de detección temprana. } \\
\text { - Edad aproximada de detección del } \\
\text { TEA. } \\
\text { - El proceso diagnóstico del TEA. } \\
\text { - Edad aproximada de diagnóstico del } \\
\text { TEA. } \\
\text { - Formación y atención de los/as } \\
\text { profesionales. }\end{array}$ \\
\hline $\begin{array}{c}\text { Atención del TEA en Centros } \\
\text { Educativos }\end{array}$ & 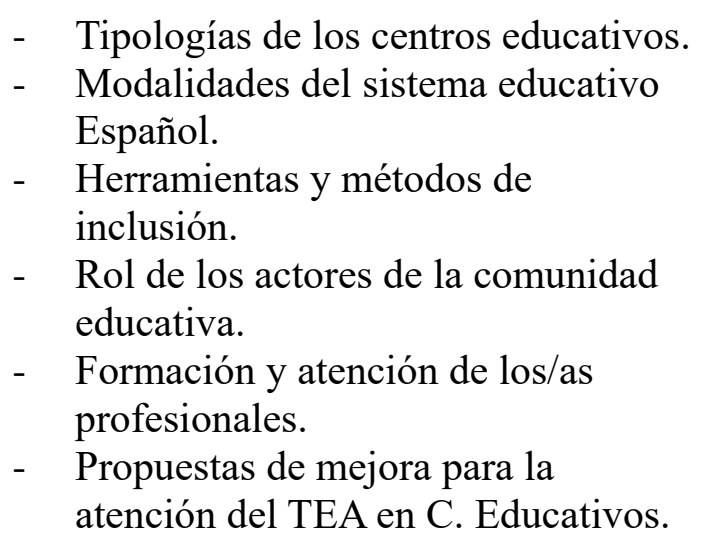 \\
\hline
\end{tabular}

La tercera fase, supone el acercamiento con las personas que desean participar, para ello hay que ejecutar un proceso de captación de sujetos que sean viables y que se adapten a las características del estudio. Se planteó realizar dos grupos de discusión, en las provincias de Jaén y Salamanca. La muestra de participantes de esta investigación, se ha establecido mediante muestreo intencional. Es un procedimiento que permite seleccionar los casos característicos de la población limitando la muestra a estos. Se utiliza en situaciones en las que la población es muy variable y consecuentemente la muestra es muy pequeña.

Para la selección de la muestra, se ha contactado con las organizaciones y entidades de TEA de la provincia de Jaén y Salamanca. Estas organizaciones informaron a sus profesionales e indicaron aquellas personas que estaban interesadas en participar en este estudio. Todos los participantes fueron informados de la duración de esta técnica, del lugar de reunión, de la fecha y el horario. Al inicio del grupo de discusión, se informa brevemente de la dinámica general de la técnica, se ofrecieron las 
pautas e indicaciones necesarias y se resolvieron las dudas e inquietudes. Conformes con todo, cada participante recibió las instrucciones por escrito y firmaron un consentimiento informado para formalizar su compromiso de participación y para consentir la grabación de imágenes, con fines exclusivos a facilitar el posterior análisis de los datos recabados por parte de los investigadores.

En la provincia de Jaén, participaron cinco profesionales de la Asociación Asperger-TEA Jaén y una profesional de un gabinete privado de psicología infantil. El grupo de discusión se constituyó por una psicóloga-terapeuta de ámbito privado, así como por miembros de la citada Asociación, participando: La directora técnica, licenciada en psicología y neuropsicología, una trabajadora social, una psicóloga, una maestra de pedagogía terapéutica y una licenciada en psicopedagogía. En concreto este grupo de discusión se desarrolló en la Unidad de Medios Audiovisuales de la Universidad de Jaén.

En la provincia de Salamanca, se optó por seleccionar a los/as profesionales a través del Centro de Atención Integral de Autismo "Infoautismo" de la Universidad de Salamanca, que está integrado en el Instituto Universitario de Integración en la Comunidad "INICO", el cual conforma una unidad docente con un carácter investigador, formativo y asistencial que responde al interés que demanda la sociedad para mejorar la calidad de la atención para todo el colectivo y sus familiares.

Se constituyó un grupo de discusión con diferentes perfiles disciplinares en la atención al TEA; participando una psicóloga, una trabajadora social, una maestra de audición y lenguaje, una terapeuta ocupacional y un maestro de educación especial. Este grupo de discusión se celebró en las instalaciones del Centro de Atención Integral "Infoautismo" en la Universidad de Salamanca.

Realizados los dos grupos de discusión, comienza la cuarta fase, que está dedicada al análisis e interpretación de la información obtenida en la técnica. Para ello, ha sido imprescindible la transcripción del discurso y las intervenciones de los participantes, con el fin de extraer y analizar los datos que han permitido establecer las conclusiones.

La quinta fase, sería mostrar los resultados de la investigación a través de un informe detallado que recoja las intervenciones y discursos de las personas que han participado en el grupo de discusión. Para concluir, lo ideal es realizar la sexta fase que consiste en validar la investigación para dar rigurosidad al trabajo.

\section{Resultados}

El estudio sobre el análisis de la atención de los menores con TEA en los centros educativos, ha sido una investigación que ha utilizado como metodología la técnica de grupos de discusión, por lo que los resultados que se han obtenido han sido únicamente cualitativos, derivados de la transcripción de los discursos obtenidos de los/as profesionales en las grabaciones de videocámara y de audio que filmaron y registraron el acto.

El primer tema que generó resultados, fue en torno a la detección temprana del TEA. Los profesionales no encuentran una edad en concreto en la que se pueda manifestar evidentes comportamientos que induzcan a sospechar que se trata de un TEA, pues varía dependiendo del menor. 
Los padres sí suelen decir que a partir de los 12 meses empiezan a encontrar cosas extrañas en sus niños, por ejemplo que no les miran o no responden a su nombre. (Profesional $\mathrm{n}^{\circ}$ 8, Grupo de Discusión 2). En el primer o segundo año de vida es cuando más se suelen ver sospechas de TEA. Por ejemplo en el juego simbólico, a los 2 años los niños empiezan a jugar, saben cómo se utiliza un cochecito o involucran a sus iguales o a la familia a que jueguen y los niños con TEA no suelen hacer eso, son más solitarios (Profesional $\mathrm{n}^{\circ} 1$, Grupo de Discusión 1). El niño con autismo puede avanzar y de repente puede tener un retroceso o ni siquiera se dan los elementos de comunicación a la edad que tienen que aparecer, entre los 12 y 18 meses (Profesional $\mathrm{n}^{\circ}$ 6, Grupo de Discusión 2). Depende un poco del nivel de TEA, porque hay algunos que se sospechan a unos 2 años y otros que se ven a los cuatro, cinco o seis años que es cuando empiezan a darse más las diferencias y se ven que se separan más del desarrollo normal (Profesional 2, Grupo de Discusión 1).

Ante estas sospechas, los familiares inician un proceso de consultas donde comunican los comportamientos de sus menores a distintos profesionales. Pero ¿Existe una figura profesional o centro $u$ organismo especializado donde dirigirse? Los profesionales que participaron en los grupos de discusión concuerdan que por regla general habría que dirigirse a un pediatra, pero ven que todavía no es un recorrido fácil.

¿Qué deben hacer los padres? Pues ojala se supiera lo que deben hacer, porque al final lo que hay es un recorrido sin sentido: por salud mental, por pediatras, por psicólogos, con neuropediatra en Córdoba, con salud mental aquí en Jaén, con psiquíatras en Sevilla....y al final nunca nadie les dice donde tienen que ir. (Profesional $\mathrm{n}^{\mathrm{o}}$ 2, Grupo de Discusión 1). Primero deberían acudir al pediatra, que es el profesional que va a abrir el camino para derivar a otros servicios (como neuropediatría), pero la mayoría de los pediatras; según me dicen las familias, yo que trabajo todos los días con las familias; les dicen pues que el niño ya hablará con el tiempo, que cada niño tiene un desarrollo diferente. Es decir hay un montón de trabas, que muchas veces son estas las barreras que hay para que no se detecte antes, porque los pediatras muchas veces les dicen que esperen a que crezca para ver como evoluciona, siempre dicen que entra dentro de la normalidad. (Profesional n ${ }^{\circ}$ 7, Grupo de Discusión 2)

La edad en la que se detecta el TEA difiere mucho con respecto a la edad en la que se inicia el proceso de diagnóstico y la fecha en la cual se obtiene un primer diagnóstico.

Lo esencial es que la familia tenga suerte y que den con un profesional que lo valore, ya puede ser un pediatra, un neuropediatra o un cuidador, tienen que tener esa suerte de que alguien de "el salto". También es verdad que si no es un pediatra no se va a "mojar" en diagnosticar, un cuidador de la guardería aunque sospeche quizás se calla. (Profesional $\mathrm{n}^{\circ}$ 5, Grupo de Discusión 2). Lo ideal sería que si el niño no tiene lenguaje, detectarlo a los 18 meses como mucho, y si tiene lenguaje que no pasara de los 3 años y eso es muy difícil. (Profesional $\mathrm{n}^{\mathrm{o}}$ 2, Grupo de Discusión 1). En Infoautismo, estamos llevando a cabo un programa de detección precoz, se llama M-CHAT y se le proporciona a los pediatras para cuando vayan a revisión los niños en torno a los 18 o 24 meses. Es como un cuestionario, con 20 items, y entonces pueden saltar items sospechosos y nos remiten los casos a nosotros, nos ponemos en contacto con la familia y si fallan los items y consideramos que el cuestionario es sospechoso 
pues se inicia una valoración. Entonces de esta manera estamos intentando bajar el nivel de diagnóstico por que a partir de los 18 meses o así, determinados items como el de la comunicación no se consigue, y si no se consigue pues a lo mejor es que hay un problema en el desarrollo, ya sea autismo, sea retraso madurativo, etc. (Profesional $n^{\circ} 5$, Grupo de Discusión 2).

En la práctica, más o menos los menores obtienen un diagnóstico a los 3 años de edad (Profesional $\mathrm{n}^{\circ}$ 9, Grupo de Discusión 2). El niño más pequeño que hemos diagnosticado en Infoautismo tenía 20 meses (Profesional $\mathrm{n}^{\mathrm{o}}$ 7, Grupo de Discusión 2).

Con respecto al proceso de diagnóstico, influye bastante la información que disponen los familiares del menor y la formación de los profesionales que atienden a estos menores.

Las familias no conocen los Centros Base y muchas de las familias que vienen aquí (infoautismo) lo detectaron leyendo en la sala de espera del centro de salud un cartel sobre las cosas que no hacen los niños autistas y no porque realmente se lo haya dicho nadie, ni haciendo las consultas periódicas, así que yo creo que las familias no tienen información. (Profesional $n^{\circ} 7$, Grupo de Discusión 2). La información la tienen poco accesible a veces, por ejemplo: Aquí en Salamanca hay dos asociaciones sobre TEA y no son todo lo accesible que tendrían que ser para las familias. Están solo destinadas a los socios, por lo que si no eres socio no puedes acceder a los servicios y esto es un problema, porque hay muchos que se quedan fuera... entonces esas familias ¿A qué recursos acuden? No los hay, para ellos no los hay, si además tienen pocos recursos económicos. (Profesional $\mathrm{n}^{\circ}$ 6, Grupo de Discusión 2). La desinformación de no saber exactamente qué es lo que tiene, qué es lo que le pasa, o cómo pueden enfrentarme a esa situación, es lo que les hace también no afrontar el diagnostico. (Profesional $\mathrm{n}^{\circ} 4$, Grupo de Discusión 1).

El problema está en que los profesionales sabemos que sí que existe el TEA, pero no sabemos identificarlo. (Profesional $\mathrm{n}^{\circ}$ 4, Grupo de Discusión 1). La clave está en la formación de los profesionales, si se forma bien a los profesionales que son los que tienen que ver los signos de alerta de autismo, antes se derivaría y todo se agilizaría. (Profesional no 9, Grupo de Discusión 2).

La etapa educativa, es una de las más importantes en el desarrollo del menor, ya que de no haberse detectado con anterioridad el TEA, es en este momento y en los centros educativos donde hay más posibilidades de diagnosticar un trastorno, debido a que es el organismo donde los menores permanecen más tiempo. En el caso de disponer de un diagnóstico precoz, ya se puede intervenir adecuadamente conforme las necesidades que presente cada menor.

En cuanto a las tipologías de los centros educativos, los profesionales que han participado en los grupos de discusión, no mantienen un acuerdo en cuanto a las diferencias que pueden existir en la atención de menores con TEA dependiendo si son centros públicos, privados y/o concertados. Aunque si bien es cierto que se declinan por los centros públicos como aquellos que pueden ofertar mejores garantías de calidad en la educación.

Realmente cualquier centro es bueno, independientemente de su característica pública o privada, dependiendo de los profesionales que tenga. (Profesional $\mathrm{n}^{\circ}$ 7, Grupo de Discusión 2). Si están formados en autismo pues las familias tienen 
esa suerte, pero si no lo están el niño no va a evolucionar tanto. (Profesional $\mathrm{n}^{\circ}$ 9, Grupo de Discusión 2). Hay diferencias en general, depende mucho de los colegios. La verdad que a nivel público, si no le proporcionan un recurso, se le puede exigir, se puede "pelear", es decir, se puede hablar con el responsable, se puede hablar con las asociaciones para que medien, pero en un privado es más dificil, al fin y al cabo en un privado la atención que reciben es la que les quieran dar, es la que puedan pagar los familiares. (Profesional $\mathrm{n}^{\circ} 2$, Grupo de Discusión 1). En los centros educativos privados realmente sólo está la figura del orientador que hace el papel de todo, de Pedagogo Terapeuta (PT) y de Audición y Lenguaje (AL). Sin embargo en los centros públicos, ya si se encuentran las figuras de los 3 profesionales (orientador, PT y AL) entonces quieras que no son tres personas que atienden al menor desde distintas perspectivas y eso de alguna manera contribuye. (Profesional $n^{\circ}$ 6, Grupo de Discusión 2).

Por otro lado, en cuanto a las distintas modalidades educativas que ofrece el Sistema Educativo Español, sucede lo mismo, no hay un consenso establecido entre los/as profesionales que han participado sobre cuál es la modalidad más idónea para los/as menores con TEA.

Depende de los casos, pero lo mejor es que el niño reciba su apoyo dentro del aula ordinaria con sus compañeros, es decir la modalidad combinada. El error de los colegios, es que siempre nos focalizamos más en los aspectos curriculares, pero es que hay muchas más cosas importantes, como enseñar habilidades sociales, emocionales, que son los aspectos que se dejan un poco de lado. (Profesional $\mathrm{n}^{\mathrm{o}}$ 4, Grupo de Discusión 1). Son tan diferentes las modalidades que a lo mejor si están bien planteadas que sean combinada, especial y ordinaria, pero muchas veces en el aula especial están chicos que deberían estar en la modalidad combinada, y ahí está el fallo, que no se sabe valorar bien. Quizás están bien valorados pero no se les envía bien a la modalidad que realmente necesitan. (Profesional $n^{\circ}$ 9, Grupo de Discusión 2). La mayoría de los casos deberían de estar en un aula ordinaria, y si precisa de algún apoyo, pues facilitárselo (Profesional $\mathrm{n}^{\circ}$ 2, Grupo de Discusión 1). Hay veces que la educación especial es un poco el cajón desastre, cuando no llegan curricularmente a lo ordinario van a educación especial, pero no es lo mismo el trato o la estructuración que tiene que tener un niño con autismo que otro que tiene una parálisis cerebral. (Profesional n 5 , Grupo de Discusión 2).

Las aulas específicas de autismo, también parece que es lo mejor según las leyes, pero en la realidad no se cumple, se deben hacer cosas con la comunidad, pero nunca llegan a hacer nada. Todo lo hacen en el aula específica, si hacen alguna excursión los llevan al huerto del colegio, pero la interacción con otros niños siempre es con los mismos compañeros que son los que están también en el aula específica. (Profesional $\mathrm{n}^{\circ}$ 2, Grupo de Discusión 1). El trabajo que hacen en las aulas específicas yo creo que es insuficiente, yo soy de las que piensa que no solo se puede quedar en esa intervención, necesitan más horas de terapia, yo creo que están recomendadas mínimo 20 horas semanales. (Profesional n ${ }^{\circ}$ 1, Grupo de Discusión 1).

En algunos casos, las modalidades educativas que ofrecen los centros educativos más que integrar y brindar los apoyos y recursos que cada menor necesita está 
provocando una segregación del alumnado. Por ello es muy importante y se hace necesario disponer de ciertas herramientas y promover métodos de inclusión.

Para la inclusión, lo primero sería cambiar el "chip" del profesor, cambiando su manera de intervenir con los niños, no siendo tan teóricos, ser más interactivos con la clase y proponer cosas más dinámicas, que no se centren en seguir un libro y poner los ejercicios. La clave está en la actitud del profesional que al final eso es lo que se contagia a los demás niños y al resto de los profesores. (Profesional $\mathrm{n}^{\mathrm{o}}$ 4, Grupo de Discusión 1). Empezando desde infantil y proporcionando mucha formación no solo a los profesionales sino también a los niños, a los compañeros del menor con TEA. Ahora por ejemplo, una de las cosas que sí que ahora sale en muchos medios de comunicación es que igual que se trabaja el día de la Paz, el día de la madre y etc. Sí que se está trabajando el día del autismo en el colegio, entonces sí que se conciencia. Pues en eso consiste, incluirlo en temas trasversales, para que vean sus compañeros que también existen niños que son especiales. (Profesional $n^{\circ}$ 6, Grupo de Discusión 2).

Los métodos que proponen los profesionales para promover la inclusión, son técnicas innovadoras y de fácil implantación por su bajo coste de implementación.

Consiste en enseñar a los compañeros cómo tratar y cómo ver el autismo de una manera diferente. (Profesional $n^{\circ}$ 5, Grupo de Discusión 2). Se deben hacer más aprendizajes cooperativos, y sobre todo no ver al niño con TEA como un niño con un problema, porque si ellos como profesores lo ven como un problema el resto de la clase, de sus compañeros también lo van a ver como un problema. Tenemos casos, de niños que han estado en su colegio muy mal y han cambiado a otro centro y al mes han estado adaptados y muy incluidos, hasta el punto de que después por la tarde sus amigos iban a su casa para jugar. (Profesional $\mathrm{n}^{\circ}$ 2, Grupo de Discusión 1).

Se utilizan prácticas como las del compañero tutor que es como una figura de referencia que hay para el menor con TEA, como por ejemplo que entre alguien para organizar los recreos y establecer juegos, por ejemplo podría ser la figura del integrador social que sí que existe. (Profesional no 7, Grupo de Discusión 2).

En cuanto a los roles de los actores de la comunidad educativa, los participantes concuerdan que lo importante es disponer de las figuras profesionales más necesarias para la atención de alumnos/as con TEA, como la Pedagogía Terapéutica, Psicología, Logopedia, Audición y Lenguaje y monitores de apoyo.

Mientras más profesionales mejor: Logopeda, pedagogo, terapeuta, psicólogo, integrador sensorial. Todo lo que pueda aprender el niño de cada profesional es bueno, aunque tampoco es cuestión de que el menor tenga un exceso de profesionales, sino que cada niño en concreto tiene unas necesidades que se deben de cubrir. (Profesional $\mathrm{n}^{\circ}$ 4, Grupo de Discusión 1). Lo importante es que el centro disponga de estos recursos humanos, pero siempre con la debida coordinación, no es cuestión que cada uno trabaje independiente. Porque si un niño está atendido por cinco profesionales y cada uno trabaja y se centra en su campo y no se coordina con el resto, al final va ser una locura. (Profesional $\mathrm{n}^{\circ}$ 6, Grupo de Discusión 2).

Pero el hecho de disponer de los recursos humanos de cada uno de los perfiles disciplinares, no garantiza una adecuada atención al alumnado con TEA. Durante el 
grupo de discusión, se abordó el tema de la formación y capacitación de los profesionales que atienden a los menores con TEA. Los participantes expusieron, la acreencia que existe en cuanto a la formación en TEA pero al mismo tiempo resaltaron que cada vez más se encuentran profesionales que tienen motivación por formarse en TEA y se preocupan por la situación.

La formación es imprescindible, porque si no la tienes sí que es verdad que no tienes herramientas para saber cómo afrontarlo. (Profesional $\mathrm{n}^{\circ} 6$, Grupo de Discusión 2). La formación del profesional, depende de cada uno, porque cada profesional puede tener su carrera y después profundizar en una formación en concreto, así que depende de la persona. (Profesional n 4, Grupo de Discusión 1). Conforme van teniendo experiencias, al final van aprendiendo, aunque en el caso del TEA cada niño es diferente y las formas de aprendizaje son diferentes por lo que no podemos llegar a extrapolarlo del todo estas experiencias, pero el hecho tener experiencias es positivo. (Profesional no 7, Grupo de Discusión 2). Es curioso que también hay casos de profesionales muy formados pero muy poco seguros de lo que están haciendo y también hemos visto a profesionales que están poco formados y que se ven segurísimos de lo bien que lo están haciendo. De hecho una vez un PT me dijo "por favor te puedes venir un día entero y observarme" y estuve yo alli las 6 horas mirándole y cuando me pregunto que cómo lo había hecho, le dije:;Genial!. Entonces lo que sucede es que muchos profesores que son buenos necesitan esa dosis de decirles que lo están haciendo bien. (Profesional n ${ }^{\circ}$, Grupo de Discusión 1)

Para finalizar, se invitó a los participantes de los grupos de discusión a que expusieran su opinión sobre cómo mejorar la atención de los menores con TEA en los Centros Educativos; y estas fueron algunas de sus propuestas:

Fomentando más las prácticas alternativas de trabajo y pedagógicas, ampliando a metodologías que sean más manipulativas. Por ejemplo hay un colegio que solo trabaja con proyectos, no con libros y eso es muy bueno para los niños con TEA, porque es más funcional (Profesional $\mathrm{n}^{\circ} 4$, Grupo de Discusión 1). Empleando más métodos de apoyos visuales y manipulativos y hacer adaptaciones curriculares pero para todo el grupo, que sean comunes, y se evite discriminar, así de esta manera pues pasa todo como más desapercibido (Profesional n ${ }^{\circ}$ 6, Grupo de Discusión 2).

Se mejoraría con más formación, con buenos profesionales y con un seguimiento de la práctica docente (Profesional $\mathrm{n}^{\circ} 3$, Grupo de Discusión 1). Implantando leyes en las que si los profesores no cumplen en la atención de los menores con TEA, pierdan su empleo. Eso no existe en ningún sitio, básicamente porque son funcionarios. (Profesional $\mathrm{n}^{\circ}$ 2, Grupo de Discusión 1). Habría que poner un sistema de calidad en la educación, basado en opiniones de padres, en opiniones de niños, basado en instituciones y basado en varios aspectos, porque si muchas veces se puede dar el caso de que hay y existe el recursos, pero... ¿El profesional le está dando la atención a ese niño que lo necesita? (Profesional $\mathrm{n}^{\circ}$ 4, Grupo de Discusión 1). 


\section{Discusión y conclusiones}

Tras la exposición de los resultados de este estudio, en el que se ha constatado que existen evidentes deficiencias en el diagnóstico temprano del TEA y habiendo mostrado que la atención que reciben los/as menores con TEA es susceptible de considerables mejoras, se puede concluir que es necesario plantearse una serie de retos a efectuar en los procesos de detección temprana y en los métodos de intervención educativa.

Sin lugar a dudas, la detección precoz del TEA es la clave fundamental para conseguir en los centros educativos una mayor inclusión y mejores estrategias de intervención. Como indicaba Attwood (1993) los TEA pueden ser diagnosticados en menores de dieciocho meses, pero en la práctica esto puede ser difícil de lograr. En este estudio se ha hecho evidente también está dificultad, ya que los profesionales que han participado tan sólo conocen un caso de un menor que lo pudieron diagnosticar a los 20 meses. Los participantes apuntan que lo más común es que los menores obtengan un diagnóstico a los tres años de edad, tal y como señalaban Hernández, J. M y cols. (2005) que indicaban que la mayoría de las ocasiones se accede a un diagnóstico final a los dos años y medio después de haber iniciado las consultas.

Es evidente que la complejidad y la naturaleza del desorden de los TEA, es una de las principales causas que retrasa su diagnóstico, pues como afirma la Federación de Autismo de Castilla y León (2006) un 62,5\% de las familias tienen dificultades en la realización del diagnóstico. Ante este hecho los participantes del grupo de discusión proponen que es necesario que los profesionales tengan mayor formación con el fin de que sepan reconocer los signos de alerta del TEA y diagnostiquen antes, pues como ellos mismos han expresado, en el proceso de diagnóstico depende mucho de la suerte o azar que el menor haya tenido en cuanto al contacto con los profesionales, y si estos estaban lo suficientemente formados como para reconocer el trastorno.

En cuanto a la atención de los menores con TEA en los centros educativos, los principales resultados de este estudio son muy similares a sus precedentes, pues todavía en la actualidad este colectivo carece de una plena inclusión educativa y siguen teniendo las mismas necesidades.

Por ejemplo, Rodríguez, I. R., Moreno, F. J. y Aguilera, A. (2007) en su estudio de los centros educativos de Sevilla, encontraron que un $73,2 \%$ de la muestra recibía los apoyos en las aulas especiales, y que este variaban dependiendo del menor, entre 1 hora y 36 horas semanales. Siendo lo más común que los/as menores recibieran sesiones de logopeda o de audición y lenguaje, entre media hora o un máximo de 5 horas semanales.

En este estudio los participantes exponen que, más allá de las horas de intervención, que a modo general siempre son insuficientes puesto que las familias lo deben cumplimentar con apoyo externo del ámbito privado y/o de las asociaciones, lo importante es que los centros educativos dispongan de los recursos humanos, que garanticen una adecuada coordinación y que evite las intervenciones independientes de cada profesional. De todo ello, se puede concluir por unanimidad que es necesario ampliar la plantilla de profesionales en los centros educativos, así como su formación y especialización en TEA tanto en los procesos de detección y diagnóstico del TEA como en la intervención educativa.

La principal limitación que ha encontrado este estudio ha sido la dificultad para acceder a los centros educativos con el fin de observar directamente la atención que 
reciben los menores con TEA. Los/as profesionales de entidades sociales, que han participado en los grupos de discusión, a través de su discurso han constatado también esta limitación ya que ellos como institución, en la mayoría de los casos no han podido acceder y colaborar con los centros educativos, siendo su única intención la de mejorar el proceso de enseñanza de los/as menores.

En posteriores y futuras investigaciones, se pretende abordar este estudio a nivel nacional, ampliando el número de los/as participantes que tienen relación de alguna manera con la población con TEA así como sus perfiles profesionales y las instituciones a las que pertenecen.

\section{Referencias}

American Psychiatric Association (2013). DSM: Manual diagnóstico y estadístico de los trastornos mentales.

Attwood T. (1993). Unusual behaviours associated with autism. Health Visitor, 66(11), 402-403.

Autism Society of America, INC. AND (2011). Autism Society of America Foundation, INC.

Consorcio de Autismo (2011). Propuesta de Plan Estratégico para las personas con trastorno de espectro autista y sus familias.

Federación Autismo Castilla y León (FACYL) (2006). Proyecto de investigación: La accesibilidad al sistema sanitario de las personas con TEA.

Grupo de Estudio de los trastornos del Espectro Autista IIER/ISCIII. (2004). Encuestas realizadas a familiares de personas con autismo en España: Informe sobre Demora Diagnóstica en los TEA. Madrid: Obra Social Cajamadrid.

Hernández, J.M. y cols. (2005). Guía de buena práctica para la detección temprana de los trastornos del espectro autista. Revista de Neurología, 41(4), 237-245.

Huertas E. y Vigier F. (2010). El grupo de discusión como técnica de investigación en la formación de traductores: Dos casos de su aplicabilidad. Revista Entreculturas, 2, 181- 196.

Ley 9/1999, de 18 de noviembre, de Solidaridad en la Educación.

Ley Orgánica 2/2006, de 3 de Mayo, de Educación.

Rodríguez, I. R., Moreno, F. J. y Aguilera, A. (2007). La atención educativa en el caso del alumnado con trastornos del espectro autista. Revista de Educación, 344, 425-445.

Suárez, M. (2005). El grupo de discusión: una herramienta para la investigación cualitativa. Barcelona: Laertes.

Fecha de recepción: 14/10/2017

Fecha de revisión: $31 / 10 / 2017$

Fecha de aceptación: 13/03/2018 